\title{
Influence des deux composantes de la vitesse (fréquence et longueur des foulées) sur le métabolisme énergétique musculaire chez le poney
}

\author{
JP Valette*, E Barrey, R Wolter \\ Laboratoire de physiologie sportive INRA, École nationale vétérinaire, \\ 7, avenue du Général-de-Gaulle, 94704 Maisons-Alfort Cedex, France
}

(Reçu le 10 avril 1990, accepté le 3 septembre 1990)

\begin{abstract}
Résumé - La vitesse de propagation d'un animal est le produit de la longueur des foulées (LF) par leur fréquence $(F F)$. Chez le poney, la production de lactate sanguin et l'élévation de la fréquence cardiaque ( $F C$ ) sont plus dépendants de $F F$ que de $L F$. Ce sont, en effet, les mêmes $F F$ qui produisent 4 mmol- $\left.\right|^{-1}$ de $L A$ et induisent une $F C$ de 200 batt- $\mathrm{min}^{-1}$, que l'effort se déroule sur le plat ou sur un tapis roulant incliné. Les vitesses seuils $\left(V_{4}\right.$ et $\left.V_{200}\right)$ sont inférieures avec un plan incliné. II en est déduit que la cadence est le facteur limitant de l'effort chez le.poney.
\end{abstract}

tapis roulant / lactatémie / fréquence cardiaque / fréquence des foulées / longueur des foulées

Summary - Influence of the two velocity components, stride frequency (SF) and stride length (SL) on muscular energetic metabollsm in the pony. Six ponies were trained for endurance over a 6-month period. They were tested twice on a treadmill, the first time with a $0 \%$ slope and the second time with a $3.5 \%$ slope. The aim of this study was to determine which component of stride (fig 1), stride length (SL) or stride frequency (SF), was more related to the lactate threshold at $4 \mathrm{mmol}^{-1}$ and the cardiac threshold at 200 beats $\cdot \mathrm{min}^{-1}$. Exercice parameters were interpolated by linear regression (table I). The slope (tables II and III) induced a decrease in the $\mathrm{V}_{4}$ and $\mathrm{V}_{200}$ values, but not in the $\mathrm{SF}_{4}$ or $\mathrm{SF}_{200}$ values. The $\mathrm{SL}_{4}$ and $\mathrm{SL}_{200}$ values were better correlated with $\mathrm{V}_{4}$ and $\mathrm{V}_{200}$ (table $\mathrm{V}$ ). It was then deduced that in the pony SF seemed to better explain the production of lactate and the increase in heart rate, and was the limiting factor in exercise.

treadmill / blood lactate / heart rate / stride frequency / stride length

\section{INTRODUCTION}

Au cours de la locomotion sportive, la quantité d'énergie nécessaire et sa vitesse d'utilisation par le muscle squelettique dépendent du type d'exercice imposé
: traction, course sur terrain accidenté. Le coût en oxygène de tels efforts (Thornton et al, 1987) a pu être estimé à partir de tests sur tapis roulants, inclinés ou non, de même que les réponses métaboliques musculaires et circulatoires (Gottlieb et al, 1988). Ces auteurs observent un accrois-

\footnotetext{
* Correspondance et tirés à part
} 
sement de la consommation d'oxygène, de la fréquence cardiaque et de la lactatémie dans les exercices d'intensité croissante en traction ou sur un plan incliné (Thornton, 1985). Ils ne constatent aucun changement dans la longueur et la fréquence des foulées qui sont les 2 composantes de la vitesse de progression. Pour accroître sa vitesse dans une même allure, le cheval augmente simultanément ces 2 composantes (Dalin et Jeffcott, 1985; Leach, 1987), mais chacune d'elles a son propre mode d'évolution (fig 1). Au fur et à mesure que la vitesse s'élève, la foulée s'allonge de façon proportionnelle, tandis que la cadence progresse de plus en plus faiblement pour atteindre une valeur maximale. Ainsi, pour un cheval sain, aux allures lentes (pas et trot détendu), la fréquence contribue davantage que l'amplitude à produire de la vitesse puis, progressivement, aux allures rapides (trot soutenu et galop), c'est la longueur qui prédomine (Barrey et al, 1989).

Le but de notre étude a été d'analyser les rapports entre le mode locomoteur adopté par l'animal et son adaptation métabolique à un effort imposé. Pour cela, nous avons recherché l'influence de l'inclinaison du tapis roulant sur les paramètres locomoteurs (fréquence et longueur des foulées) et energétiques (production d'acide lactique et évolution de la fréquence cardiaque).

\section{MATÉRIEL. ET MÉTHODES}

Six poneys de conformations differentes (140$220 \mathrm{~kg}$ ) sont entrainés en aérobiose, à $65 \%$ de leur fréquence cardiaque maximum (130-140 batt $/ \mathrm{min}$ ), 3 fois par semaine pendant 6 mois au moyen d'une longe automatique. Les épreuvestests ont lieu sur tapis roulant après $10 \mathrm{~min}$ d'échauffement au trot. La première à plat, la seconde avec une pente de $3,5 \%$, afin d'accroître le travail locomoteur demandé.

L'épreuve-test comporte 6 paliers de vitesses croissantes (de 2-7 m/s), adaptées à chaque animal afin que les 2 derniers paliers s'effectuent à une fréquence cardiaque supérieure à $200 \mathrm{batt} / \mathrm{min}$. Chaque palier d'effort dure $3 \mathrm{~min}$ avec un intervalle de repos de 2 min au cours duquel est effectuée une prise de sang. L'acide lactique, après déprotéinisation avec $\mathrm{HClO}_{4} 0,6$ $N$ est dosé au moyen d'une électrode spécifique (LM2 analyser, Analox Instruments). La fréquence cardiaque ( $F C$ ) est enregistrée en continu au moyen d'un cardio-fréquencemètre (Horse Tester PEH 200, Polar Electro). La fréquence des foulées (FF) est déterminée d'après le chronométrage du temps écoulé pendant $\mathbf{4 0}$ poses du même membre par le rapport suivant :

\section{$F F[\mathrm{~Hz}]=40 /$ temps}

La longueur de la foulée (LF) en est déduite par la relation :
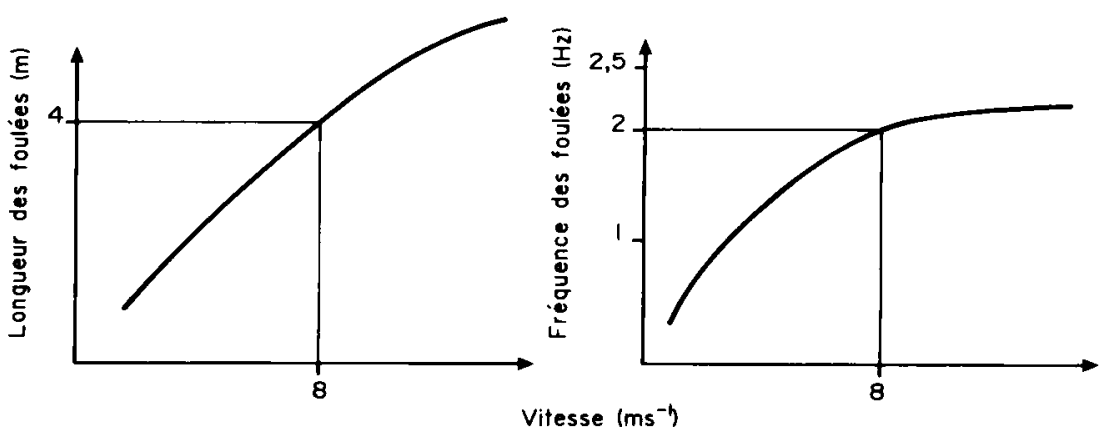

Fig 1. Evolution de la longueur et de la fréquence des foulées en fonction de la vitesse. Vitesse = longueur des foulées $\mathrm{x}$ fréquence des foulées. 


$$
L F[m]=\text { vitesse } / F F
$$

L'étude des relations entre vitesse $(V)$, lactatémie (La), FC, FF, LF définit un certain nombre de critères physiologiques (tableau I) qui évaluent l'aptitude à l'effort (Valette et Wolter, 1988). Ces relations sont établies par régression linéaire après, en particulier, transformation logarithmique de la réponse lactique. La vitesse $V i(\mathrm{~m} / \mathrm{s})$ choisie comme vitesse de référence, correspond à la vitesse médiane du test.

Les résultats ont été traités par le test $t$ de Student (cas des séries appariées) et par l'étude des corrélations.

\section{RÉSULTATS}

Le tableau II récapitule les valeurs individuelles des 6 poneys lors des 2 épreuves.

Le tableau III rapporte les moyennes observées lors des 2 épreuves tests. Les valeurs $V_{4}, L F_{4}, V_{200}, L F_{200}$ baissent de manière significative avec la pente, alors que FFVi augmente. Les fortes variations des valeurs de $p L F$ et de $p F F$ ne sont pourtant pas significatives en raison du comportement hétérogène des animaux vis-à-vis de la pente.

L'étude des corrélations (tableau IV, $r 10 \mathrm{ddl}=0,58)$ entre les variables nous montre que, au seuil conventionnel de $4 \mathrm{mmol} / \mathrm{l}$, la vitesse $\left(V_{4}\right)$ est mieux corrélée avec la longueur de la foulée $\left(L F_{4}\right.$, $r=0,84$ ) qu'avec la fréquence des foulées $\left(F F_{4}, r=0,60\right)$. A une puissance d'exercice induisant 200 batt cardiaques/min $\left(V_{200}\right)$, seule la longueur de la foulée $\left(L F_{200}\right)$ est corrélée $(r=0,94)$. Les liaisons qui existent entre $L F_{4}$ et $L F_{200}(r=0,97)$ comme entre $F F_{4}$ et $F F_{200}(r=0,84)$ découlent de celle qui unit $V_{4}$ et $V_{200}(r=0,91)$. LFVi est corrélée avec $L F_{4}(r=0,91)$ et $L F_{200}$ $(r=0,93)$; quant à $F F V i$, elle est liée avec $L F_{4}(r=-0,92)$ et $L F_{200}(r=-0,94)$, mais paradoxalement, ni avec $F F_{4}(r=0,11)$, ni avec $F F_{200}(r=0,27)$.

\section{DISCUSSION}

L'introduction d'une pente lors d'une épreuve d'effort sur tapis roulant augmente le travail locomoteur demandé à l'animal.

Tableau I. Définitions des critères d'aptitude à l'effort.

\begin{tabular}{lll} 
Relation & Paramètre & Définition \\
\hline La vs vitesse & $V_{4}$ & Vitesse produisant une lactatémie de $4 \mathrm{mmol} / 1$ \\
$F C$ vs vitesse & $V_{200}$ & Vitesse induisant une $F C$ de $200 \mathrm{batt} / \mathrm{min}$ \\
$L F$ vs vitesse & $L F V i$ & $L F$ correspondant à la vitesse $V i(4,5 \mathrm{~m} / \mathrm{s})$ \\
$F F$ vs vitesse & $F F V i$ & $F F$ correspondant à la vitesse $V i(4,5 \mathrm{~m} / \mathrm{s})$ \\
Lactate vs FF & $F F_{4}$ & $F F$ produisant une lactatémie de $4 \mathrm{mmol} /$ \\
Lactate vs FF & $L F_{4}$ & Corollaire de $F F_{4}$ \\
$F C$ vs $F F$ & $F F_{200}$ & $F F$ induisant une $F C$ de \\
$F C$ vs $L F$ & $L F_{200}$ & 200 batt $/ \mathrm{min}$
\end{tabular}


Tableau II. Paramètres synthétiques calculés pour les 6 poneys lors des 2 épreuves tests.

\begin{tabular}{|c|c|c|c|c|c|c|c|c|c|}
\hline Poneys & $F F V i$ & $L F V i$ & $V_{4}$ & $F_{4}$ & $L F_{4}$ & $V_{200}$ & $F F_{200}$ & $L F_{200}$ & Périodes \\
\hline \multicolumn{10}{|c|}{ Epreuve test $n^{\circ} 1$} \\
\hline $\begin{array}{l}1 \\
2 \\
3 \\
4 \\
5 \\
6\end{array}$ & $\begin{array}{l}2,00 \\
2,48 \\
2,61 \\
2,35 \\
2,67 \\
2,14\end{array}$ & $\begin{array}{l}2,19 \\
1,78 \\
1,70 \\
1,87 \\
1,65 \\
2,08\end{array}$ & $\begin{array}{l}6,20 \\
5,55 \\
5,00 \\
5,30 \\
5,10 \\
5,30\end{array}$ & $\begin{array}{l}2,25 \\
2,73 \\
2,72 \\
2,56 \\
2,81 \\
2,26\end{array}$ & $\begin{array}{l}2,83 \\
2,05 \\
1,81 \\
2,08 \\
1,81 \\
2,39\end{array}$ & $\begin{array}{l}7,20 \\
5,80 \\
5,00 \\
5,80 \\
5,25 \\
5,20\end{array}$ & $\begin{array}{l}2,42 \\
2,79 \\
2,74 \\
2,69 \\
2,87 \\
2,26\end{array}$ & $\begin{array}{l}3,28 \\
2,12 \\
1,85 \\
2,20 \\
1,90 \\
2,40\end{array}$ & $\begin{array}{l}1,00 \\
1,00 \\
1,00 \\
1,00 \\
1,00 \\
1,00\end{array}$ \\
\hline \multicolumn{10}{|c|}{ Epreuve test $n^{\circ} 2$} \\
\hline $\begin{array}{l}1 \\
2 \\
3 \\
4 \\
5 \\
6\end{array}$ & $\begin{array}{l}2,07 \\
2,56 \\
2,82 \\
2,50 \\
2,75 \\
2,53\end{array}$ & $\begin{array}{l}2,12 \\
1,75 \\
1,60 \\
1,79 \\
1,64 \\
1,72\end{array}$ & $\begin{array}{l}4,75 \\
3,95 \\
1,60 \\
4,10 \\
3,30 \\
3,85\end{array}$ & $\begin{array}{l}2,11 \\
2,35 \\
1,67 \\
2,40 \\
2,47 \\
2,32\end{array}$ & $\begin{array}{l}2,20 \\
1,65 \\
1,35 \\
1,69 \\
1,31 \\
1,58\end{array}$ & $\begin{array}{l}6,05 \\
4,00 \\
3,05 \\
4,40 \\
3,40 \\
4,40\end{array}$ & $\begin{array}{l}2,44 \\
2,37 \\
2,29 \\
2,48 \\
2,50 \\
2,60\end{array}$ & $\begin{array}{l}2,67 \\
1,65 \\
1,27 \\
1,77 \\
1,35 \\
1,77\end{array}$ & $\begin{array}{l}2,00 \\
2,00 \\
2,00 \\
2,00 \\
2,00 \\
2,00\end{array}$ \\
\hline
\end{tabular}

* Les corrélations comportant un astérisque en exposant sont signicatives au seuil $P<0,05$.

Les modifications énergétiques sont observables à 2 puissances métaboliques particulières, $V_{4}$ et $V_{200}$. Les indices $V_{4}$ et $V_{200}$ sont traditionnellement utilisés comme indicateurs de la forme physique (Persson, 1983; Wilson et al, 1983) et sont des estimateurs traditionnels, quoique biaisés (Valette et al, 1989), du seuil anaérobie. En présence d'une pente, les poneys atteignent une lactatémie de $4 \mathrm{mmol} / \mathrm{l}$ et une fréquence cardiaque de $200 \mathrm{batt} / \mathrm{min}$ pour des vitesses $\left(V_{4}\right.$ et $\left.V_{200}\right)$ inférieures à

Tableau III. Résultats des épreuves tests.

\begin{tabular}{lllll}
\hline Critères & Test 1:plat & Test 2: pente & T5ddl & Seuil \\
\hline FFVi & $2,38 \pm 0,24$ & $2,54 \pm 0,24$ & 3,2 & 0,05 \\
LFVi & $1,88 \pm 0,20$ & $1,77 \pm 0,17$ & 2,1 & NS \\
$V_{4}$ & $5,4 \pm 0,40$ & $3,6 \pm 1,0$ & 5,6 & 0,01 \\
$F F_{4}$ & $2,63 \pm 0,22$ & $2,45 \pm 0,1$ & 2,1 & NS \\
$L F_{4}$ & $2,16 \pm 0,36$ & $1,63 \pm 0,29$ & 8,1 & 0,001 \\
$V_{200}$ & $5,7 \pm 0,75$ & $4,2 \pm 0,95$ & 8,0 & 0,001 \\
$F F_{200}$ & $2,56 \pm 0,22$ & $2,22 \pm 0,27$ & 1,5 & NS \\
$L F_{200}$ & $2,29 \pm 0,48$ & $1,75 \pm 0,46$ & 16,8 & 0,001 \\
\hline
\end{tabular}


Tableau IV. Corrélations entre les variables $(n=12 ; r=0,58)$

\begin{tabular}{lrrllllll}
\hline & $F F V i$ & $L F V i$ & $V_{4}$ & $F F_{4}$ & $L F_{4}$ & $V_{200}$ & $F F_{200}$ & $L F_{200}$ \\
\hline$F F V i$ & 1,00 & & & & & & & \\
$L F V i$ & $-0,99^{*}$ & 1,00 & & & & & & \\
$V_{4}$ & $-0,70^{*}$ & $0,64^{*}$ & 1,00 & & & & & \\
$F F_{4}$ & 0,11 & $-0,20$ & $0,61^{*}$ & 1,0 & & & \\
$L F_{4}$ & $-0,92^{*}$ & $0,91^{*}$ & $0,84^{*}$ & 0,08 & 1,00 & & \\
$V_{200}$ & $-0,83^{*}$ & $0,78^{*}$ & $0,91^{*}$ & 0,31 & $0,93^{*}$ & 1,00 & \\
$F F_{200}$ & 0,27 & $-0,37$ & 0,40 & $0,84^{*}$ & $-0,07$ & 0,27 & 1,00 & \\
$L F_{200}^{200}$ & $-0,94^{*}$ & $0,93^{*}$ & $0,81^{*}$ & 0,04 & $0,98^{*}$ & $0,95^{*}$ & $-0,06$ & 1,00 \\
& & & & & & & & \\
\hline
\end{tabular}

- Les corrélations comportant un astérisque en exposant sont significatives au seuil de $P<0,05$.

celles obtenues lors des exercices sur une surface horizontale. Des résultats analogues ont été trouvés chez le cheval (Thornton et al, 1987).

A puissances métaboliques comparables $\left(V_{4}\right.$ et $\left.V_{200}\right)$, la fréquence des foulées à ces vitesses particulières $\left(F F_{4}\right.$ et $\left.F F_{200}\right)$ ne varie pas de façon significative, alors que la longueur des foulées $\left(L F_{4}\right.$ et $\left.L F_{200}\right)$ baisse $(P<0,001)$. Ceci démontre que la production d'acide lactique et l'élévation de la fréquence cardiaque sont dépendantes de la cadence chez le poney, puisque ce sont à peu près les mêmes tréquences qui produisent $4 \mathrm{mmol}^{-1}$ de lactate et induisent une $F C$ de $200 \mathrm{batt} / \mathrm{min}^{-1}$, que l'effort se déroule sur le plat ou avec une inclinaison du tapis roulant. Chez le trotteur, Thornton et al (1987) ne notent aucune modification de $\mathrm{FF}_{4}$ et de $L F_{4}$ avec une inclinaison de $6,25 \%$, avec ou sans chargement du cheval.

A vitesse comparable $(V i=4,5 \mathrm{~m} / \mathrm{s})$, l'inclinaison pourtant faible du tapis roulant induit une augmentation significative de la fréquence de la foulée et, en corrolaire, l'animal diminue l'amplitude de sa foulée.
Chez le poney, la fréquence des foulées atteint des valeurs plus élevées (valeurs maximales observées de l'ordre de 2,7-2,9 foulées/s) que chez le cheval (environ 2,22,3 foulées/s). Ces résultats sont en accord avec le modèle de Heglund et al (1974), qui met en évidence la relation entre la taille de l'animal et la cadence adoptée par celui-ci aux différentes allures. Comme l'ont montré les travaux de Attenburrow (1982), la fréquence respiratoire est exactement couplée à la fréquence des foulées au galop. Les résultats présentés ici montrent également l'influence de la cadence sur la dépense énergétique. Ces observations expérimentales sembleraient indiquer que ce paramètre locomoteur est un facteur limitant de l'effort de puissance chez le poney.

\section{REMERCIEMENTS}

Ce travail a été subventionné par l'INRA, département de pathologie animale et par le service des haras. 


\section{RÉFÉRENCES}

Attenburrow DP (1982) Time relation between the respiratory cycle and limb in the horse. Equine Vet $J 14,69-72$

Barrey E, Francqueville A, Barrey JC, Demonceau $T(1989 b)$ Cadence et amplitude des foulées. Equathion 1 (4), 6-14

Dalin G, Jeffcott LB (1985) Locomotion and gait analysis. Vet Clin N Am Equine Pract 1, 549572

Gottlieb M, Essen-Gustavssonn B, Lindholm A, Persson SGB (1988) Circulatory and muscle metabolic responses to draught work compared to increasing trotting velocities. Equine Vet $J 20,430-434$

Heglund NC, Taylor CR, McMahon TA (1974) Scaling stride frequency and gait to animal size: mice to horse. Science 186, 1112-1113

Leach DH (1987) Locomotion of the athletic horse. In: Equine Exercise Physiology II. ICEEP Publ, Davis, Ca, 516-535
Persson SGB (1983) Evaluation of exercise tolerance and fitness in the performance horses. In: Equine Exercise Physiology. Granta, Cambridge, 441-457

Thornton JR (1985) Exercise testing. Vet Clin N Am Equine Pract 1, 573-595

Thornton JR, Pagan J, Persson SGB (1987) The oxygen cost of weight loading and inclined treadmill exercise in the horse. in: Equine Exercise Physiology II. ICEEP Publ, Davis, $\mathrm{Ca}, 206-215$

Valette JP, Wolter $R$ (1988) Intérêt des mesures de lactatémie et de fréquence cardiaque comme critères d'aptitudes sportives. CEREOPA, 14e journée d'étude, 9 mars, $1-16$

Valette JP, Barrey E, Garbasi C, Wolter R (1989) Estimation du seuil anaérobie chez le poney. Ann Zootech 38, 229-236

Wilson RGF, Isler RB, Thornton JR (1983) Heart rate, lactic acid production and speed during a standardized exercise test in standardbred horses. In: Equine Exercise Physiology. Granta, Cambridge, 487-496 